BULL. AUSTRAL. MATH. SOC.

VOL. $21(1980), 47-63$.

\title{
ON THE SPECTRUMY OF STEIN QUASIGROUPS
}

\author{
F.E. Bennett and N.S. Mendelsohn
}

\begin{abstract}
In this paper we investigate the spectrum of a variety of quasigroups satisfying the 2-variable identity $x(x y)=y x$, called Stein quasigroups. Stein quasigroups are known to be selforthogonal and have been given a considerable amount of attention because of this property. It is known that there are no stein quasigroups of order $2,3,6,7,8,10,12,14$. The object of this paper is to show that for all but 36 values of $n \geq 15$ there exists a Stein quasigroup of order $n$. In particular, the spectrum of Stein quasigroups contains all $n \geq 191$.
\end{abstract}

\section{Introduction}

The problem of determining the spectrum of quasigroups satisfying the identity $x(x y)=y x$ was raised by Stein in [14]. Stein [14] and Mendelsohn [8] used Galois fields to obtain such quasigroups of order $4^{k} m$, where the square-free part of $m$ does not contain any prime $p \equiv 2$ or $3(\bmod 5)$. In [15], Stein used balanced incomplete block designs (see Hanani [5]) to construct quasigroups of orders $12 k+1$, $12 k+4,20 k+1,20 k+5$. Lindner [7] further enlarged the spectrum by using the singular direct product of Sade [13]. More recently, Pelling and Rogers [10] used pairwise balanced designs in conjunction with the singular direct product to show that stein systems exist for all orders $n \geq 1042$. In [11], they also showed that orders $2,3,6,7,8,10,12$, 14 are impossible and investigated certain varieties of Stein systems.

In this paper, we further enlarge the spectrum of Stein systems

Received 3 January 1979. A portion of this work was done at the 1978 Séminaire de Mathématiques Supérieures, Université de Montréal. The authors wish to thank A.E. Brouwer for some helpful suggestions. 
studied in [10], [11]. It is shown that for all but 36 values of $n \geq 15$ there exists a stein system of order $n$. In particular, Stein systems exist for all orders $n \geq 191$. Our method of construction is similar to that employed in [10]. However, much credit is owed to Brouwer, whose constructions in [2] helped to establish the main lemmas of Section 3.

\section{Preliminaries}

The following information on designs will be quite useful in most of our constructions and the reader is referred to Hanani [5].

DEFINITION 2.1. Let $K$ be a set of positive integers. A pairwise balanced design of index unity $B(K, 1 ; v)$ is a pair $(X, B)$, where $X$ is a $v$-set (of points) and $B$ is a collection of subsets of $X$ (called blocks) with sizes in $K$ such that every pair of distinct points of $X$ is contained in exactly one block of $B .|X|$ is called the order of the pairwise balanced design.

DEFINITION 2.2. Let $K$ and $M$ be sets of positive integers. A group divisible design $G D(K, 1, M ; v)$ is a triple $(X, G, B)$, where

(i) $X$ is a v-set (of points),

(ii) $G$ is a collection of non-empty subsets of $X$ (called groups) with sizes in $M$ and which partition $X$,

(iii) $B$ is a collection of subsets of $X$ (called blocks), each with size at least two in $K$,

(iv) no block meets a group in more than one point, and

(v) each pairset $\{x, y\}$ of points not contained in a group is contained in exactly one block.

If all groups $g \in G$ have size $|g| \geq 2$, then a group divisible design $(X, G, B)$ is a pairwise balanced design $(X, G \cup B)$ in which a collection of blocks which partition $X$ has been distinguished. We shall write $B(k, 1 ; v)$ for $B(\{k\}, 1 ; v)$ and similarly, $G D(k, 1, m ; v)$ for $G D(\{k\}, 1,\{m\} ; v)$. Note that a star appearing on an element of $K$. (or $M$ ) means that there is exactly one block (or group) of that size in the pairwise balanced design (or group divisible design). We observe that a pairwise balanced design $B(k, 1 ; v)$ is a balanced incomplete block design with parameters $v, k$, and $\lambda=1$. 
DEFINITION 2.3. A transversal design $T(k, 1 ; m)$ is a group divisible design $G D(k, 1, m ; k m)$, where each block is a transversal of the collection of groups.

DEFINITION 2.4. Let $(X, B)$ be a pairwise balanced design $B(K, 1 ; v)$. A paralzel class in $(X, B)$ is a collection of disjoint blocks of $B$, the union of which equals $X .(X, B)$ is called resolvable if the blocks of $B$ can be partitioned into parallel classes. A group divisible design $G D(K, 1, M ; v)$ is resolvable if its associated pairwise balanced design $B(K \cup M, 1 ; v)$ is resolvable with $M$ as a parallel class of the resolution.

It is known [5] that the existence of a resolvable transversal design $R T(k, 1 ; m)$ is equivalent to the existence of a transversal design $T(k+1,1 ; m)$. It is also fairly well-known [4] that the existence of a $T(k, 1 ; m)$ is equivalent to the existence of a set of $k-2$ mutually orthogonal latin squares of order $m$. We state with references some of the fundamental results which will be used in subsequent sections.

THEOREM 2.5. A resolvable design $R B(3,1 ; v)$, called a Kirkman triple system, exists if and only if $v \equiv 3(\bmod 6)$ (see [12]).

THEOREM 2.6. A design $B(4,1 ; v)$ exists if and only if $v \equiv 1$ or $4(\bmod 12)($ see $[5])$.

THEOREM 2.7. A resolvable design $R B(4,1 ; v)$ exists if and only if $v \equiv 4(\bmod 12) \quad($ see $[6])$.

THEOREM 2.8, A design $B(5,1 ; v)$ exists if and only if $v \equiv 1$ or $5(\bmod 20)($ see $[5])$.

THEOREM 2.9. A transversal design $T(5,1 ; m)$ exists for all positive integers $m$ with the exception of $m=2,3,6$ and possibly excepting $m=10$ and 14 (see [5], [9], [16]).

The following theorem has been used extensively in investigating the spectrum of quasigroup models of certain collections of 2-variable identities. As in [10], it forms the basis of our paper.

THEOREM 2.10. Let $V$ be a variety of algebras which is idempotent and which is based on 2-variable identities. Suppose there is a pairwise balanced design $B(K, 1 ; v)$ such that for each $k \in K$ there is a model of $V$ of order $k$, then there is a model of $V$ of order $v$. 
At this stage, we point out that Stein quasigroups are necessarily idempotent and self-orthogonal ([3]). Thus Theorem 2.10 will be very much applicable in our constructions and the results can be interpreted in terms of self-orthogonal Latin squares. However, it is known [1] that there are self-orthogonal Latin squares of all orders $n \neq 2,3,6$.

\section{The main lemmas}

The auxiliary results of this section are based mainly on the use of resolvable designs and transversal designs (see [2]). We shall let $B(K)$ denote the set of integers $v$ for which a pairwise balanced design $B(K, 1 ; v)$ exists. Unless otherwise stated, a starred element of $K$ means that there is a unique block of that size in the pairwise balanced design $B(K, I ; v)$.

Suppose $(X, B)$ is a resolvable design (pairwise balanced design) on $v$ points, where $B=\bigcup_{i=1}^{r} B_{i}$ is a partition of $B$ into parallel classes. If $1 \leq m \leq r$, we may obtain a design on $v+m$ points by adding to $X$ a set $S=\left\{a_{1}, a_{2}, \ldots, a_{m}\right\}$ of $m$ new points. We then replace each block $b \in B_{i}$ by $b \cup\left\{a_{i}\right\}$ for $I \leq i \leq m$ and take $S$ as a block of size $m$. By applying this technique to a resolvable design $R B(3,1 ; 6 k+3)$ (Kirkman triple system), which has $3 k+1$ parallel classes, and a resolvable design $R B(4,1 ; 12 k+4)$, which has $4 k+1$ parallel classes, we readily obtain the following two lemmas.

LEMMA 3.1. If $k>1$, then $9 k+4 \in B(4,(3 k+1) *)$.

LEMMA 3.2. If $1 \leq m \leq 4 k+1$, then $12 k+m+4 \in B\left(4,5, m^{*}\right)$, where, in case $m=4$ or 5 , the star means that a block of size $m$ is distinguished while all other blocks are of size 4 or 5 .

The following lemma is proved in [2].

LEMMA 3.3. A pairwise balanced design $B\left(\left\{4,7^{*}\right\}, 1 ; v\right)$ exists if and only if $v \equiv 7$ or $10(\bmod 12), v \neq 10,19$.

If we start with a transversal design $T(5,1 ; m)$ and delete $m-n$ points from a particular group, assuming $0 \leq n \leq m$, we obtain a group divisible design $G D(\{4,5\}, 1,\{m, n\} ; 4 m+n)$, called a truncated 
transversal design (see [5]). In view of Theorem 2.9, we can state the following useful result.

LEMMA 3.4. If $m \neq 2,3,6,10,14$ and $0 \leq n \leq m$, then there exists a group divisibie design $G D(\{4,5\}, 1,\{m, n\} ; 4 m+n)$.

From Lemma 3.4, we obtain immediately

LEMMA 3.5. If $m \neq 2,3,6,10,14$ and $0 \leq n \leq m$, then $4 m+n+1 \in B(4,5, m+1, n+1)$.

Proof. We take the blocks of a $G D(\{4,5\}, 1,\{m, n\} ; 4 m+n)$ and each group with a fixed additional point adjoined.

More generally we have

LEMMA 3.6. Suppose $m \neq 2,3,6,10,14$ and $0 \leq n \leq m$. Then we have

(i) $4 m+n+r \in B(4,5, m+r)$, if $m+r \in B\left(4,5, r^{*}\right)$ and $n+r \in B\left(4,5, r^{*}\right)$,

(ii) $4 m+n+r \in B(4,5, n+r)$, if $m+r \in B\left(4,5, r^{*}\right)$, where in case $r=4$ or 5 , the star means that a block of size $r$ is distinguished while all other blocks are of size 4 or 5 .

Proof. Let $(X, G, B)$ be the $G D(\{4,5\}, I,\{m, n\} ; 4 m+n)$ of Lemma 3.4. Let $g_{1}, g_{2}, g_{3}, g_{4}$ be the four groups of size $m$ and let $g_{5}$ be the group of size $n$ in $G$. Let $S$ be a set of $r$ fixed points disjoint from $X$. We add these points to the group divisible design. In order to prove $(i)$, we construct a $B(\{4,5, m+r\}, 1 ; 4 m+n+r)$ on $X \cup S$ as follows:

(1) take the blocks of $B$,

(2) replace the group $g_{5}$ by a $B\left(\left\{4,5, r^{*}\right\}, 1 ; n+r\right)$ on the set $S \cup g_{5}$ which has $S$ among its blocks,

(3) for $i=1,2,3$, replace each group $g_{i}$ by a $B\left(\left\{4,5, r^{*}\right\}, 1 ; m+r\right)$ on the set $S \cup g_{i}$ with $S$ among its blocks, and finally, 


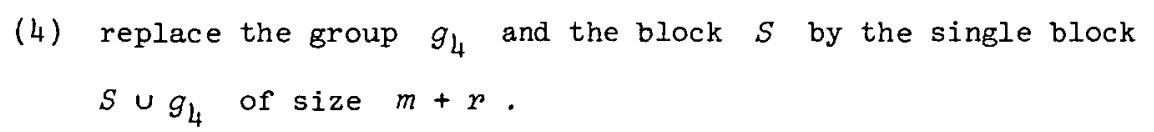

The result is a $B(\{4,5, m+r\}, 1 ; 4 m+n+r)$ and $(i)$ is proved. In order to prove $(i i)$, we construct a $B(\{4,5, n+r\}, 1 ; 4 m+n+r)$ by taking the blocks of $B$, then we replace the groups $g_{i}, 1 \leq i \leq 4$, by a $B\left(\left\{4,5, r^{*}\right\}, 1 ; m+r\right)$ on the set $S \cup g_{i}$ which has $S$ among its blocks and finally, we replace the group $g_{5}$ and the block $S$ by the single block $S \cup g_{5}$ of size $n+r$. This completes the proof of the lemma.

As an application of Lemma 3.6, we state the following two important results.

LEMMA 3.7. If $1 \leq s \leq k-1$, then

$$
48 k+12 s+10 \in B(4,5,(12 k+7) *) \text {. }
$$

Proof. We choose $m=12 k, n=12 s+3$, and $r=7$ in Lemma 3.6 . By Lemma $3.3,12 k+7 \in B\left(4,7^{*}\right)$ for all $k \geq 2$ and $12 s+10 \in B\left(4,7^{*}\right)$ for all $s \geq 1$, and the result follows immediately from Lema 3.6 (i).

LEMMA 3.8. If $1 \leq s \leq k$, then

$$
48 k+12 s+22 \in B\left(4,5,(12 k+10)^{*}\right) \text {. }
$$

Proof. We choose $m=12 k+3, n=12 s+3$, and $r=7$ in Lemma 3.6. By Lemma 3.3, $12 k+10 \in B\left(4,7^{*}\right)$ for all $k \geq 1$ and $12 s+10 \in B\left(4,7^{*}\right)$ for all $s \geq 1$. The result follows from Lemm 3.6 (i).

The technique of adding a set of fixed points to a truncated transversal design will be quite instrumental in most of our constructions. Some special cases of importance are the following.

LEMMA 3.9. If $1 \leq s \leq k$, then $48 k+12 s+26 \in B\left(4,5,11^{*}\right)$.

Proof. We choose $m=12 k+4$ and $n=12 s+4$ in Lemma 3.4 , and let. $(X, G, B)$ be the $G D(\{4,5\}, 1,\{12 k+4,12 s+4\} ; 48 k+12 s+20)$. Let $g_{1}, g_{2}, g_{3}, g_{4}$ be the four groups of size $12 k+4$ and let $g_{5}$ be the group of size $12 s+4$ in $G$. Let $b=\left\{a_{1}, a_{2}, a_{3}, a_{4}, a_{5}\right\}$ be a block of $B$ of size 5 where $b \cap g_{i}=\left\{a_{i}\right\}, I \leq i \leq 5$. Now we add to the 
group divisible design a set $S$ of size 6 disjoint from $X$. We construct a $B\left(\left\{4,5,11^{*}\right\}, 1 ; 48 k+12 s+26\right)$ on $X \cup S$ as follows:

(1) take the blocks $B-\{b\}$,

(2) for $i=1,2,3,4$, replace each group $g_{i}$ by a $B\left(\left\{4,7^{*}\right\}, 1 ; 12 k+10\right)$ on the set $S \cup g_{i}$ with $S \cup\left\{a_{i}\right\}$ as its unique block of size 7 ,

(3) replace the group $g_{5}$ by a $B\left(\left\{4,7^{*}\right\}, 1 ; 12 s+10\right)$ on the set $S \cup g_{5}$ with $S \cup\left\{a_{5}\right\}$ as its unique block of size 7 , and finally,

(4) replace the five blocks $s \cup\left\{a_{i}\right\}, 1 \leq i \leq 5$, by the single block $S \cup b$ of size 11 .

It is readily checked that the result is a $B\left(\left\{4,5,11^{*}\right\}, 1 ; 48 k+12 s+26\right)$ and the lemma is proved.

Similarly, we have

LEMMA 3.10. If $1 \leq s \leq k-1$, then $48 k+12 s+14 \in B\left(4,5,11^{*}\right)$.

Proof. We choose $m=12 k+1$ and $n=12 s+4$ in Lemma 3.4 and starting with the $\operatorname{GD}(\{4,5\}, 1,\{12 k+1,12 s+4\} ; 48 k+12 s+8)$, we add a set of 6 new points. By using the existence of a $B\left(\left\{4,7^{*}\right\}, 1 ; 12 k+7\right)$ for $k \geq 2$ and $a\left(\left\{4,7^{*}\right\}, 1 ; 12 s+10\right)$ for $s \geq 1$ together with the technique used in the proof of Lemma 3.9, we may construct a $B\left(\left\{4,5,11^{*}\right\}, 1 ; 48 k+12 s+14\right)$ to obtain the desired result.

Since Lemma 3.2 guarantees the existence of a $B\left(\left\{4,5,7^{*}\right\}, 1 ; 12 k+11\right)$ for all $k \geq 2$, we may further obtain

LEMMA 3.11. If $k \geq 2$ and $1 \leq s \leq k$, then $48 k+12 s+30 \in B\left(4,5,11^{*}\right)$.

Proof. We choose $m=12 k+5$ and $n=12 s+4$ in Lemma 3.4 and let $(X, G, B)$ be the $G D(\{4,5\}, 1,\{12 k+5,12 s+4\} ; 48 k+12 s+24)$. Let $g_{1}, g_{2}, g_{3}, g_{4}$ be the four groups of size $12 k+5$ and let $g_{5}$ be the group of size $12 s+4$ in $G$. Let $b=\left\{a_{1}, a_{2}, a_{3}, a_{4}, a_{5}\right\}$ be a block of $B$ of size 5 where $b \cap g_{i}=\left\{a_{i}\right\}$. We add to the group divisible 
design a set $S$ of size 6 disjoint from $X$ and construct a $B\left(\left\{4,5,11^{*}\right\}, 1 ; 48 k+12 s+30\right)$ on $X \cup S$ as follows:

(I) take the blocks $B-\{b\}$,

(2) for $i=1,2,3,4$, replace each group $g_{i}$ by a $B\left(\left\{4,5,7^{*}\right\}, 1 ; 12 k+11\right)$ on the set $S \cup g_{i}$ with $S \cup\left\{a_{i}\right\}$ as its unique block of size 7 ,

(3) replace the group $g_{5}$ by a $B\left(\left\{4,7^{*}\right\}, 1 ; 12 s+10\right)$ on the set $S \cup g_{5}$ with $S \cup\left\{a_{5}\right\}$ as its unique block of size 7 , and finally,

(4) replace the five blocks $S \cup\left\{a_{i}\right\}, 1 \leq i \leq 5$, by the single block $S \cup b$ of size 11 .

The resulting design is a $B\left(\left\{4,5,11^{*}\right\}, 1 ; 48 k+12 s+30\right)$ and the lemma is proved.

The following lemma is a consequence of Theorem 2.8.

LEMMA 3.12. If $n \equiv 1$ or $5(\bmod 20)$ and $k=1,4$, or 5 , then $n-k \in B(4,5)$.

Proof. We take a $B(5,1 ; n)$ and delete $k$ points which are contained in the same block. The result follows.

\section{Existence theorems}

In what follows we shall use the notation of [10] and write $R(v)$ if there exists a Stein system of order $v$. In order to establish the main result, the following well-known theorem (see [8], [14]) will be used in conjunction with the results of the previous sections.

THEOREM 4.1. $R(p)$, if $p$ is a prime congruent to $0,1,4(\bmod 5)$, and $R\left(p^{2}\right)$, if $p$ is a prime congruent to $2,3(\bmod 5)$.

REMARK 4.2. For our purposes, it will suffice to note that Theorem 4.1 guarantees $R(n)$ for $n=4,5,9,11,19$, and 31 . If we can show that $v \in B(4,5,9,11,19,31)$, then it follows from Theorem 2.10 that $R(v)$ holds. It is important to note that in most of our constructions we tacitly use the fact that if $v \in B\left(K^{\prime}\right)$ and $K^{\prime} \subseteq B(K)$, then $v \in B(K)$. 
THEOREM 4.3. If $v \equiv 0$ or $1(\bmod 4)$ and $v \neq 8,12$, then $v \in B(4,5,9,11)$ and $R(v)$.

Proof. If $k \geq 1$ and $v=12 k+1,12 k+4,12 k+5,12 k+8,12 k+9$, then $v \in B(4,5)$ follows from Theorem 2.6 and Lemma 3.2. If $k \geq 5$, then $12 k+24 \in B\left(4,5,20^{*}\right)$ by Lemma 3.2. Consequently, $12 k+24 \in B(4,5)$ for $k \geq 5$. If $v=24,36,60$, then $v \in B(4,5)$ follows from Lemma 3.12 . For the cases $v=48$ and 72 , we apply Lemma 3.4 as follows: $48=4.11+4 \in B(4,5,11)$, and $72=4.17+4 \in B(4,5,17) \subseteq B(4,5)$. This completes the proof of the theorem.

THEOREM 4.4. If $v \equiv 3(\bmod 12)$ and $v \neq 3,15,27,39$, then $v \in B(4,5,11)$ and $R(v)$.

Proof. If $k \geq 3$, then, by Lemma 3.2, $12 k+15 \in B\left(4,5,11^{*}\right)$ and the result follows.

THEOREM 4.5. If $v \equiv 11(\bmod 12)$ and $v \neq 23,35$, then $v \in B(4,5,11,19)$ and $R(v)$.

Proof. If $k \geq 5$, then $12 k+23 \in B\left(4,5,19^{*}\right)$ follows from Lemma 3.2. In addition, we have

$$
\begin{aligned}
& 47=(4.9+7)+4 \in B(4,5,11) \text { by Lemma } 3.6(i i), \\
& 59=(4.12+7)+4 \in B(4,5,11) \text { by Lemma } 3.6(i i), \\
& 71=(4.15+10)+1 \in B(4,5,11) \text { by Lemma } 3.5 .
\end{aligned}
$$

This completes the proof of the theorem.

THEOREM 4.6. If $v \equiv 7(\bmod 12)$ and $v \neq 7,43$, then $v \in B(4,5,11,19,31)$ and $R(v)$.

Proof. If $k \geq 13$, then $12 k+55 \in B(4,5,11)$ follows from Lemma 3.2 and the fact that $51 \in B(4,5,11)$ by Theorem 4.4. Thus, for $v \equiv 7$ (mod 12) and $v \geq 211$, we have $v \in B(4,5,11)$. If $k \geq 2$, then $(4(12 k-1)+10)+1 \in B(4,5,11)$ follows from Lemma 3.5 and $12 k \in B(4,5,11)$. In particular, $\{103,151,199\} \subseteq B(4,5,11)$. If $k \geq 1$, then we have

$$
\begin{aligned}
& 4(12 k+8)+11 \in B(4,5,11) \text { by Lemma } 3.4 \text { and } 12 k+8 \in B(4,5), \\
& 4(12 k+5)+11 \in B(4,5,11) \text { by Lerma } 3.4 \text { and } 12 k+5 \in B(4,5) \text {. }
\end{aligned}
$$

In particular, $\{79,91,127,139,175,187\} \subseteq B(4,5,11)$. For the cases 
$v=55,67,115,163$, we have

$$
55=5.11 \in B(5,11) \text { by Theorem } 2.9 \text {. }
$$

$67=4.16+3 \in B(4,5,19)$ by Lerma $3.6(i)$, using $19 \in B\left(4,5,3^{*}\right)$ by Lemma 3.2 .

$115=4.28+3 \in B(4,5,31)$ by Lemma $3.6(i)$, using

$31 \in B\left(4,5,3^{*}\right)$ by Lemma 3.2 .

$163 \in B(4,5,11)$ as follows: let $(X, G, B)$ be a $G D(4,1,38 ; 152)$, which exists by Lemma 3.4. Add to the group divisible design a set $S$ of size 11 disjoint from $X$. Since we have $49=4.11+5 \in B(4,5,11)$, we may replace each group $g$ of size 38 in $G$ by a $B(\{4,5,11\}, 1 ; 49)$ on the set $S \cup g$ which has $S$ as one of its blocks and we get the desired result. This completes the proof of the theorem.

REMARK 4.7. In the proof of Theorem 4.6, we realize that $163=4.36+19 \in B(4,5,19)$. However, it is important to note that we have essentially proved that $12 k+7 \in B(4,5,11)$ for all $k \geq 4$, $k \neq 5,9$.

THEOREM 4.8. If $v \equiv 2(\bmod 12)$ and $v \neq 2,14,26,38,50,62,74,98,110,158$,

then $v \in B(4,5,11)$ and $R(v)$.

Proof. We shall apply Lemmas 3.9 and 3.10 as follows: if in Lemma 3.10 we put $s=1$, then

$$
48 k+26 \in B(4,5,11) \text { for } k \geq 2 \text {. }
$$

If in Lemma 3.9 we put $s=1,2,3$, then, respectively,

$$
\begin{aligned}
& 48 k+38 \in B(4,5,11) \text { for } k \geq 1, \\
& 48 k+50 \in B(4,5,11) \text { for } k \geq 2, \\
& 48 k+62 \in B(4,5,11) \text { for } k \geq 3 .
\end{aligned}
$$

These results guarantee $v \in B(4,5,11)$ for all $v$ stated in the theorem and the proof is complete.

THEOREM 4.9. If $v \equiv 6(\bmod 12)$ and $v \neq 6,18,30,42,54,66,78,90,102,114,126,162,174$, 
then $v \in B(4,5,11)$ and $R(v)$.

Proof. We apply Lemma 3.11 as follows: if in Lemma 3.11 we put $s=1,2,3,4$, then, respectively,

$$
\begin{aligned}
& 48 k+42 \in B(4,5,11) \text { for } k \geq 2, \\
& 48 k+54 \in B(4,5,11) \text { for } k \geq 2, \\
& 48 k+66 \in B(4,5,11) \text { for } k \geq 3, \\
& 48 k+78 \in B(4,5,11) \text { for } k \geq 4 .
\end{aligned}
$$

The above results give $v \in B(4,5,11)$ for all $v$ stated in the theorem, with the exception of $v=222$. For the case $v=222$, we first observe that $51=4.11+7 \in B\left(4,5,11,7^{*}\right)$ by Lemma 3.4 . Next we put $m=44$ and $n=39$ in Lemma 3.4 and let $(X, G, B)$ be the $G D(\{4,5\}, 1,\{44,39\} ; 215)$ obtained. Let $g_{1}, g_{2}, g_{3}, g_{4}$ be the four groups of size 44 and let $g_{5}$ be the group of size 39 in $G$. We add a block $S$ of size 7 disjoint from $X$ to the group divisible design. We replace the group $g_{5}$ by a $B\left(\left\{4,7^{*}\right\}, 1 ; 46\right)$ on the set $S \cup g_{5}$ with $S$ as a block and replace each group $g_{i}, i=1,2,3$, by a $B\left(\left\{4,5,11,7^{*}\right\}, 1 ; 51\right)$ on the set $S \cup g_{i}$ with $S$ as a block. Finally, replace the block $S$ and the group $g_{4}$ by a $B(\{4,5,11\}, 1 ; 51)$ on the set $S \cup g_{4}$. The result is a $B(\{4,5,11\}, 1 ; 222)$ on the set $X \cup S$. The proof of the theorem is complete.

THEOREM 4.10. If $v \equiv 10(\bmod 12)$ and

$$
v \neq 10,22,34,46,70,82,106,130,142,178,190
$$

then $v \in B(4,5,9,11,19,31)$ and $R(v)$.

Proof. We apply Lemma 3.7 as follows: if in Lemma 3.7 we put $s=1,2,3,4$, then respectively,

$$
\begin{array}{ll}
48 k+22 \in B(4,5,12 k+7) & \text { for } k \geq 2, \\
48 k+34 \in B(4,5,12 k+7) & \text { for } k \geq 3, \\
48 k+46 \in B(4,5,12 k+7) & \text { for } k \geq 4, \\
48 k+58 \in B(4,5,12 k+7) & \text { for } k \geq 5 .
\end{array}
$$


By Theorem 4.6, $B(4,5,12 k+7) \subseteq B(4,5,11,19,31)$ for all $k \geq 1$, $k \neq 3$. Consequently, these results guarantee $v \in B(4,5,9,11,19,31)$ for all $v$ in the statement of the theorem, with the exception of $v=58,94,154,166,202$, and 250. However, by Lemma 3.1,

$$
\begin{aligned}
& 58 \in B(4,19), \\
& 94 \in B(4,31), \\
& 166 \in B(4,55) \subseteq B(4,5,11),
\end{aligned}
$$

and

$$
202 \in B(4,67) \subseteq B(4,5,19)
$$

By Lemma 3.8,

$$
250=48.4+12.3+22 \in B(4,5,58) \subseteq B(4,5,19) .
$$

For the case $v=154$, we first observe that $22 \in B\left(4,7^{*}\right)$, and $39=(4.8+6)+1 \in B\left(4,5,9,7^{*}\right)$ by Lemma 3.5 . Next, we apply the technique used in the proof of Lemma 3.9 to add 6 new points to a $G D(\{4,5\}, 1,\{33,16\} ; 4.33+16)$ and form a $B(\{4,5,9,11 *\}, 1 ; 154)$. This completes the proof.

It is known [11] that there are no Stein systems of order 2, 3, 6, 7, $8,10,12,14$. Summarizing the results of this section, we have proved

THEOREM 4.11. $v \in B(4,5,9,11,19,31)$ for alZ $v \geq 191$.

THEOREM 4.12. $R(v)$ holds for all $v$ with the exception of $v=2,3,6,7,8,10,12,14$, and possibly excepting 15, 18, 22, 23, 26, $27,30,34,35,38,39,42,43,46,50,54,62,66,70,74,78,82,90,98$, $102,106,110,114,126,130,142,158,162,174,178,190$.

\section{Subsystems}

In this section we mention some results which extend the spectrum of certain classes of Stein systems (see [10], [11]).

If a Stein system $S$ contains a proper subsystem $T$, then $|S| \geq 3|T|+1$ (see [11]). Some cases where equality holds have been stated in [10]. In particular, if we write $Q(n)$ whenever there is a Stein system of order $n$ which is a subsystem of one of order $3 n+1$, then it was shown that $Q(n)$ holds for $n=4^{m}\left(3^{k+1}-1\right) / 2$ and $m, k \geq 0$. 
More generally, we have the following result.

THEOREM 5.1. If $n \equiv I(\bmod 3)$ and

$$
n \neq 7,10,22,34,43,46,70,82,106,130,142,178,190,
$$

then $Q(n)$ holds.

Proof. The proof follows directly from Lemma 3.1 and Theorem 4.12.

A medial Stein system is a Stein system which satisfies the medial law $(x y)(z t)=(x z)(y t)$. It is known [11] that the spectrum of medial stein systems consists of all integers whose square-free part does not contain any prime $p \equiv 2,3(\bmod 5)$. An extended medial system is a Stein system with the property that every 2-element generated subsystem is medial. It was established in [10], [11] that extended medial systems exist for all orders $n \geq 1198$. Combining Theorem 4.11 with the fact that there are medial Stein systems of orders $4,5,9,11,19$, and 31 , we immediately obtain

THEOREM 5.2. Extended medial systems exist for all orders $n \geq 191$.

Stein systems which have the property that every 2-element generated subsystem is of order 4 or 5 or 11 have been constructed in [10]. Such systems exist for all orders $v \equiv .0$ or $1(\bmod 4), v \neq 8,9,12$ (see the proof of Theorem 4.3 or Theorem 6 of [10]). For the orders $v \equiv 3(\bmod 4)$ and $v \equiv 2(\bmod 4)$, lower bounds of 247 and 1198 , respectively, have been given in [10]. In the following two theorems, these lower bounds are improved to 119 and 298 , respectively.

THEOREM 5.3. If $v \equiv 3(\bmod 4)$ and

$$
v \neq 3,7,15,19,23,27,31,35,39,43,67,83,115 \text {, }
$$

then $v \in B(4,5,11)$.

Proof. In Theorem 4.4, we proved that $12 k+15 \in B(4,5,11)$ for $k \geq 3$. In the proof of Theorem 4.6 , it is shown that $12 k+7 \in B(4,5,11)$ if $k \geq 4$ and $k \neq 5,9$. In addition, we have, by applying Lemma 3.5 , $48 k+11=(4(12 k)+10)+1 \in B(4,5,11)$ for $k \geq 1$,

and

$$
48 k+23=(4(12 k+3)+10)+1 \in B(4,5,11) \text { for } k \geq 1 .
$$

By applying Lemma 3.6 (ii), we obtain 


$$
48 k+47=(4(12 k+9)+7)+4 \in B(4,5,11) \text { for } k \geq 0 .
$$

For $k \geq 2$, we may add 6 new points to a

$$
G D(\{4,5\}, 1,\{12 k+1,25\} ; 4(12 k+1)+25)
$$

and apply the technique used in the proof of Lemma 3.9 to obtain, by using the existence of a $B\left(\left\{4,7^{*}\right\}, 1 ; 12 k+7\right)$ and a $B\left(\left\{4,7^{*}\right\}, 1 ; 31\right)$, a $B\left(\left\{4,5,11^{*}\right\}, 1 ; 48 k+35\right)$. That is, $48 k+35 \in B(4,5,11)$ for $k \geq 2$. By combining these results, it is an easy matter to check that $v \in B(4,5,11)$ for the values of $v$ stated in the theorem.

THEOREM 5.4. If $v \equiv 2(\bmod 4)$ and $v \geq 298$, then $v \in B(4,5,11)$.

Proof. If $v \equiv 2$ or $6(\bmod 12)$, Theorems 4.8 and 4.9 guarantee $v \in B(4,5,11)$ for all $v \geq 186$. If $v \equiv 10(\bmod 12)$, then Lemma 3.7 gives us

$$
\begin{array}{ll}
48 k+22 \in B(4,5,12 k+7) & \text { for } k \geq 2, \\
48 k+34 \in B(4,5,12 k+7) \text { for } k \geq 3, \\
48 k+46 \in B(4,5,12 k+7) \text { for } k \geq 4, \\
48 k+58 \in B(4,5,12 k+7) \text { for } k \geq 5 .
\end{array}
$$

By Remark 4.7, $12 k+7 \in B(4,5,11)$ for all $k \geq 4, k \neq 5,9$. Thus the results stated above imply that if $v \equiv 10(\bmod 12)$ and $v \geq 214$ and $v \neq 250,262,274,286,298,454,466,478,490$, then $v \in B(4,5,11)$.

In what follows, we show that

$$
\{166,274,298,454,466,478,490\} \subseteq B(4,5,11) .
$$

By Lemma 3.1, $166 \in B(4,55) \subseteq B(4,5,11)$ and

$274 \in B(4,91) \subseteq B(4,5,11)$. For the case $v=298$, we have

$$
79=(4.15+14)+5 \in B\left(4,5,19^{*}\right) \text { by Lema } 3.6 \text { (ii), }
$$

and

$$
58 \in B\left(4,19^{*}\right) \text { by Lemma } 3.1 .
$$

Thus, by Lemma 3.6 (i), we have

$$
298=(4.60+39)+19 \in B(4,5,79) \subseteq B(4,5,11) .
$$

By applying Lemma 3.4 and the fact that $86 \in B(4,5,11)$ by Theorem 4.8, 


$$
\begin{aligned}
& 454=4.92+86 \in B(4,5,86,92) \subseteq B(4,5,11), \\
& 466=4.95+86 \in B(4,5,86,95) \subseteq B(4,5,11),
\end{aligned}
$$

and

$$
490=4.101+86 \in B(4,5,86,101) \subseteq B(4,5,11) .
$$

By applying Lemma 3.5 , we obtain

$$
478=(4.98+85)+1 \in B(4,5,86,99) \subseteq B(4,5,11) .
$$

It is clear that if $v \equiv 10(\bmod 12)$ and $v \geq 298$, then $v \in B(4,5,11)$ and the proof of the theorem is complete.

In conclusion, we wish to consider another class of Stein systems, specifically, Stein systems which contain a subsystem of order 4 and a subsystem of order 5 . Such systems have interesting properties and applications will be given in a subsequent paper by the authors. If we write $R^{*}(v)$ whenever there exists a stein system of order $v$ which contains a subsystem of order 4 and a subsystem of order 5 , then we have the following theorem.

THEOREM 5.5. $R^{*}(v)$ holds for all $v$ with the exception of $v \quad 15$ and possibly excepting $16,18,19,22,23,25,26,27,28,30,31$, $34,35,38,39,42,43,46,50,54,55,58,62,66,70,74,78,82,90,94$, $98,102,106,110,114,126,130,142,158,162,174,178,190$.

Proof. The exceptions $v \leq 15$ result from our opening remarks on subsystems. If we combine the proofs of Theorems $4.3,4.4,4.5,4.6,4.8$, 4.9, and 4.10, then, apart from the above exceptions, the only cases where $R^{*}(v)$ is not guaranteed are $v=21,12 k+1,12 k+4$, where $k \geq 1$, in Theorem 4.3, $v=19,31,55$ in Theorem 4.6, and $v=58,94$ in Theorem 4.10. In order to complete the proof of the theorem, we shall show that $R^{*}(v)$ holds for $v=21$ and for all $v \equiv 1$ or $4(\bmod 12), v \geq 37$. By Lemma 3.12,

$$
\begin{aligned}
& 21=25-4 \in B(4,5), 37=41-4 \in B(4,5), \\
& 40=45-5 \in B(4,5), \\
& 64=65-1 \in B(4,5), 76=81-5 \in B(4,5) .
\end{aligned}
$$

By Lemma 3.4,

$$
73=4.17+5 \in B(4,5), 88=4.21+4 \in B(4,5) .
$$


By Lemma 3.5,

$$
52=(4.12+3)+1 \in B(4,5), 61=5.12+1 \in B(4,5) .
$$

By Lemma $3.6(i)$,

$$
49=5.9+4 \in B(4,5,13) \subseteq B(4,5) .
$$

Finally, by Lemma 3.2 ,

$$
12 k+25 \in B\left(4,5,21^{*}\right) \subseteq B(4,5) \text { for } k \geq 5 \text {, }
$$

and

$$
12 k+28 \in B\left(4,5,24^{*}\right) \subseteq B(4,5) \text { for } k \geq 6 \text {. }
$$

This completes the proof of the theorem.

\section{References}

[1] R.K. Brayton, Donald Coppersmith and A.J. Hoffman, "Self-orthogonal Latin squares of all orders $n \neq 2,3,6 "$, Bulz. Amer. Math. Soc. $80(1974), 116-118$.

[2] A.E. Brouwer, "Optimal packings of $\mathrm{K}_{4}$ 's into a $\mathrm{K}_{\mathrm{n}}$ " (Mathematisch Centrum Preprint ZW92/77).

[3] J. Dénes, A.D. Keedwell, Latin squares and their applications

(Akadémiai Kiadó, Budapest; English Universites Press, London; 1974).

[4] Marshall Hall, Jr., Combinatomial theory (Blaisdell [Ginn \& Co.], Waltham Massachusetts; Toronto, Ontario; London; 1967).

[5] Haim Hanani, "Balanced incomplete block designs and related designs", Discrete Math. 11 (1975), 255-369.

[6] Haim Hanani, D.K. Ray-Chaudhuri and Richard M. Wilson, "On resolvable designs", Discrete Math. 3 (1972), 343-357.

[7] Charles C. Lindner, "Construction of quasigroups satisfying the identity $x(x y)=y x$ ", Canad. Math. Buzz. 14 (1971), 57-59. 
[8] N.S. Mendelsohn, "Combinatorial designs as models of universal algebras", Recent progress in combinatorics, 123-132 (Proc. Third Waterloo Conf. Combinatorics, 1968. Academic Press, New York and London, 1969).

[9] W.H. Mills, "Three mutually orthogonal Latin squares", $J$. Combinatorial Theory Ser. A 13 (1972), 79-82.

[10] M.J. Pelling and D.G. Rogers, "Stein quasigroups I: combinatorial aspects", Buzl. Austral. Math. Soc. 18 (1978), 221-236.

[11] M.J. Pelling and D.G. Rogers, "Stein quasigroups II: algebraic aspects", Buzl. Austral. Math. Soc. 20 (1979), 321-334.

[12] D.K. Ray-Chaudhuri and Richard M. Wilson, "Solution of Kirkman's schoolgirl problem", Combinatorics, 187-203 (Proc. Symposia Pure Mathematics, 19. American Mathematical Society, Providence, Rhode Island, 1971).

[13] A. Sade, "Produit direct-singulier de quasigroupes orthogonaux et anti-abéliens", Ann. Soc. Sci. Bruxelles Sêr. I 74 (1960), 91-99.

[14] Sherman K. Stein, "On the foundations of quasigroups", Trons. Amer. Math. Soc. 85 (1957), 228-256.

[15] Sherman K. Stein, "Homogeneous quasigroups", Pacific J. Math. 14 (1964), 1091-1102.

[16] S.M.P. Wang and Richard M. Wilson, "A few more squares, II", Proceedings of the Ninth Southeastern Conference on Combinatorics, Graph Theory, and Computing, Boca Raton, Florida, 1978 (to appear).

Department of Mathematics and Astronomy,

University of Manitoba,

Winnipeg,

Manitoba,

Canada R3T 2N2. 